\section{Psychopathic disorder and autistic spectrum disorders}

Sir: As a child and adolescent psychiatrist I would like to support the observations of Gralton \& Crocombe (Psychiatric Bulletin, November 1999, 23, 692). Autistic spectrum disorders are becoming much more common and this is not just a reflection of more awareness. There has been a definite increase since 1979 in the diagnosis of children with this disorder and a very small minority of people with Asperger's syndrome do show very violent behaviour which is dangerous to others with no sense of remorse and no understanding that violence is wrong.

At present it is mainly child and adolescent psychiatrists who notice this increase, but many of these young people are now reaching adult age and will need to move on to adult services.

From our experience of working with children and young people I would very definitely feel that the service provision for individuals suffering from autistic spectrum disorders and psychopathic disorders should be quite different from provision for antisocial or dissocial personality disorders.

Linda Winkley, Consultant Child \& Adolescent Psychiatrist, Oaklands Centre, Birmingham Children's Hospital, B29 6JB

\section{CPD - Module C}

Sir: I have been in correspondence with Professor Katona and others regarding the College's Continuing Professional Development requirements and the difficulties of those of us who are neither currently in NHS posts or salaried posts in large private hospitals.

I am very pleased to see that the College wants to be as flexible as possible, for which I am personally grateful. I have been fortunate in being able to be involved in local NHS educational sessions, for which I am thankful to colleagues but I do wonder if there is any interest among those, for example, who work full-time in medico-legal work, occupational health or journalism, getting together on a regular, but reasonably infrequent basis, to enable relevant Module $C$ requirements to be met.

I would welcome hearing from anybody interested in this proposal.

Duncan A. Veasey, Consultant Psychiatrist, Rectory Farm, East Chaldon Road, Winfrith Newburgh, Nr Dorchester, Dorset DT2 8DJ

\section{Treating offenders with personality disorder}

Sir: Maden (Psychiatric Bulletin, December 1999, 23, 707-710) advocates that the concept of 'treatability' of psychopathic disorder "must go, before irreparable damage is done to psychiatry's public standing". He condemns psychiatrists who diagnose an untreatable personality disorder and conclude that supervision is impossible and leave a probation officer to carry out this supervision. By distancing ourselves from the concept of 'treatability' are we not becoming more concerned with public protection rather than treatment of patients?

Damian Mohan, Consultant Forensic Psychiatrist Broadmoor Hospital, Crowthorne, Berkshire RG45 7EG

\section{the college}

\section{Examination results}

\section{MRCPsych Part I}

\section{Examination - Autumn 1999}

The following 226 candidates were successful in the MRCPsych Part Examination held in Autumn 1999, from a total of 568 candidates who sat the Examination (pass rate: $39.79 \%$ ).

The pass rate for those candidates who sat the Clinical Examination was $71.07 \%$.

In accordance with the Examination Regulations, these candidates may now proceed to the MRCPsych Part II Examination, provided that they satisfy the requirements for entry: two-and-ahalf years (30 months) full-time postregistration approved training, a minimum of two years' registration as a medical practitioner in the United Kingdom or Republic of Ireland, and sponsorship by their Tutor and Consultant Supervisor.

Simon Adelman, Hosakere

Subrahmanyam Aditya, Nazir Ahmed, Imoukhuede Akande, Muhammad Saleem Akhtar, Akindele Olatoye Akioye, Mohamed Abdullah Ali Salih, Zaubia Alyas, Muthukumaraswamy Anandamurugan, Jennifer Mary Anderson, Sreedharan Pillai Anilkumar (Pillai), Elen Rhiannon Mair Ap Gwilym, Caroline Jane Ardron, Arcot Dhandayudham Arunmozhi, Lucinda Atkinson, Saravanan Balaji, Noreen Elizabeth Bannan, Sarah Bartlett
Joanna Michelle Beaumont, Ralf-Peter Behrendt, Michael David Beranek, Andrea Karen Brown, Anda Bude, Jayraj Burrun, Asha Butt, Neetha Byrappa, Catherine Majella Cahill, William Ross Calthorpe, John Malcolm Weatherstone Cameron, Lucy Anne Cameron, Colin Donald Campbell, Basil Silas Cardoza, Stuart Michael Carney, Matthew Zachary David Castle, Kai Tai Chan, Erika Jane Chess, David Mackinnon Barrett Christmas, Jude Nnamdi Chukwuma, Sabina Alessia Ciani, Rikus Hermanus Coetzee, Paul Robert Cohen, Gemma Majella Colhoun, Sangita Cook, Corinne Anne Corbett, Rebecca Marie Corteling, Mary-Anne Cotton, Stephanie Cozzi, Darren James Craddock, Clare Teslin Cribb, William Jonathan Cutter, Brian Joseph Manual Darnley, Eric James Davies, Simon Jonathan Cheshire Davies, Johanna Wilhelmina de Villiers, Marta Di Forti, Maria de los Angeles Diaz-Caneja, Adam Mathew Dierckx, Susan Adrianne Shealagh Dolan, Maja Dujic, Maha Ahmed Ismail El-Nadeef, Olumuyiwa John Farnoroti, Mina Susan Fazel, Jason Robert Fee, Karen Tracy Fehilly, Yolanda Helen Ferguson, Naida Frances Forbes, Dianne Elizabeth Forsyth, Karen Lisa Franks, David Glynn Gerber, Asher Reuven Giora, Geraldine Glover, Vivienne Sara Gould, Vicente Gradillas Gonzalez, Jan Henry Groszer, Sainina Gul, Gyles Vann Haden, Judith Mary Halford, Odile Patricia Hally, Zafrullah Hamzah, Dietmar Hank, Gabra
Elea Gabra Hanna, Jayaraman Hariram, Charlotte Brigitte Harris, Mahnaz Hashmi, Sarah Holmes, Rebecca Louise Horne, Oliver David Howes, Juliet Diana Hurn, Amr Salah Ibrahim Abdallah Ibrahim, Furhan Iqbal, Rebecca Jacob, Ather Sajjad Jafri, Ilan Gideon Joffe, Sheher Yar Jovindah, Amjad Adnan Khalil Jumaian, Sudhir Kaligotla, Rajagopal Keerthy Sunder, Brendan Desmond Kelly, Ann Louise Keulder, Ayla Sabrina Khan, Vivek Khosla, Olasunkanmi Bayonle Koladaisi, Prem Kumar Kunjukrishnan, Kuruvilla K Kuruvilla, Yee Tine Robert Lam, Heinrich Christiaan Lamprecht, Laval Richard Hin Wa Law Min, Gordon Patrick Lehany, Blair Peter Duncan Leslie, James Stewart Gillespie Lewis, Wei Ming Liao, Sian Annette Llewellyn-Jones, Alma Winifred Lydon, Cecelia Aoibhinn Lynch, Olive Lynn, Callum Maccall, Sheena Margaret MacKenzie, Darren James Mackintosh, Kim Caroline Maguire, Shahid Majid, Alison Jean Marr, Derek John Marshall, Thomas Marshall, Jasenka Matekovic, Olukemi Yetunde Mateola, Craig Jeffrey Mearns, Renato Merolli, Jonathan Millard, Iain Joseph Mitchell, Mirza Wasi Mohamad, Andrew Carl Molodynski, Joseph Patrick Molony, Tirthankar Mukhejee, Amer Mukhtar, Margaret Ita Mulholland, Jane Margaret Murdoch, Catriona Murray, P. J. Naga Venkatesha Murthy, Sanyogita Nadkarni, Asim Naeem, Jonathan Robert Nash, Jitendra Kumar Nayar, Harish Kumar 
Neelakant, Rajesh Baburao Nehete, Wendy Jane Neil, Ewan Graham Neilson, Allison Newman, Romanus Ndubueze Nnaji, Margaret Therese Nolan, Henry Patrick O'Connell, Sean Ciaran O'Domhnaill, Izucffukwy Sylvester Ogbata, David James Ogden, Amin Hassan Osman Omer, Sheriff Ayinde Orekan, Susan Carole Painter, Andrew James Russell Parker, Hywell Rhys Pearce, Colin Mark Peebles, Wai Phyo, Maxwell Pickard, Asha Stanley Praseedom, Vadagur Narayanappa Premachandra, Wemer Muller Pretorius, Nicholas Jeremy Price, Christine Rosalind Pugh, Parthasarathy Pushparaja Sekaran, Lammata Bala Raju, Uhich Michael Rath, Amanda Redvers, Alan Stuart Reid, Euan Thomas Smith Robertson, Halivana Siddiah Roopa, Peter James Roots, Renarta Louise Rowe, Dimitris Samellas, Helen Olutokunbo Sasegbon, Victoria Elizabeth Savage, Venkatarama Gupta Savitha Sri Eranti, Domenico Scala, Dorothea Elisabeth Schirgel, Joanna Clare Selman, Samrat Sengupta, Benjamin St John Sessa, Gadelrab Mohamed Sharief, Jagdish Singh Sidhu, Yadavindra Shah Singh, Wei Man Bonnie Siu, Daniel Joseph Smith, Meenal Milind Sohani, Oluwatoyin Ayodeji Sorinmade, Jayanth Srinivas, Ronald Garvin St Hill, Jonathan Mark Steele, Rosalind Stephenson, James Michael Stone, Ruth Audrey Sym, Eduardo Szaniecki, Lucille Margaret Taylor, Sharon Elisa Taylor, Helen Clair Temperton, Joyce Louise Theodosiou, Georgios Steliou Theodoulou, Srinavasan Thirumalai, Subhashini N. Thiyagesh, Paul Alexander Tiffin, Kristiane Brigitte Traffa, Kim Joy Ullyatt, Philippus Willem Van Driel, Hugo Alexander Van Odyck, Sathyamoorthy Vanathy, Jyoti Vandana, Pablo Vandenabeele, Maria Dolores Velazquez Guerra, Haido Marie Vlachos, Dermot Joseph Ward, Adrian Paul Warnock, Joanne Watkins, Elizabeth Maria Weich, Catherine Shân Williams, Justin Edward Howitt Wilson, Adrian Mark Winrow, Brenda Wright, Babur Zahir Yusufi, Habib Fatima Zaki, Hamid Zamani.

\section{MRCPsych Part II}

\section{Examination - Autumn} 1999

The following 178 candidates were successful in the MRCPsych Part II Examination held in Autumn 1999, from a tota of 374 candidates who sat the Examination (pass rate: $47.59 \%$ ).

In accordance with the Bye-Laws, the election of these candidates to Membership of the College and the use of the letters 'MRCPsych' after their names is dependent upon payment to the College of a registration fee, following which a certificate of Membership will be issued by the College.

Mohammed Tahib Adetokunbo Abiodun Abioye, Niruj Kumar Agrawal, Farooq Ahmad, Katina Maria Anagnostakis, Amanda Fleur Ashby, Sheikh Asif Zia, Clare Atkinson, Asif Atta, Linda Barron, Alison Jane Battersby, Daniel Matthew Beales, Jonathan Paul Damian Beckett, Carl Beuster, Irma Beyers, Seth Ayyaz Bhunnoo, Joanne Henrietta Black, Lisa Claire Blissitt, Leonie Willis Boeing, Peter Stanley Braben, John Brady, Christine Braeman, Andrew John Brindley, Joan Margaret Bufton, William Leonard Burbridge-James, Anne Barbara Cahill, Alasdhair James Caldwell, Apu Tapash Chakraborty, Cheong Fai Chan, Gloria Fong Yeung Chan, Suresh Chari, Oi Lam Chau, Koi Man Cheng, Elizabeth ClarkeSmith, Murray James Barclay Cochrane, Lucinda Mary Cockayne, Tara Louise Collinge, Andrew Thomas Collins, Ciaran Diamuid Corcoran, Justin Roger Daddow, Rajan Darjee, Sharon Davies, Rejin Dayanandan, Diana de Bruyne, Peter John Dean, Dolores Maria Del Estal Huertas, Philip Charles Dodd, Alexandra Lys Doubtfire, Dearbhla Mary Ursula Duffy, Thomas Rhys Edwards, Julie Karen Ellison, Haraldur Erlendsson, Pia Jane Feldmann, Fiona Mary Fenton, Emma Charlotte Fergusson, Rachel Filik, John Bernard Frazer, Olwyn Claire Gallagher, Claire Gallagher, Damian St. John Gamble, Ruth Auxiliadora Garcia-Rodriguez, Noel Alexander Gavin, Sobhi Ezzat Abdelnour Girgis, Susan Mary Goodwin, Mhairi Gourlay, Neil Greenberg, Joseph Michael Mirham Guirguis, Quazi Shams Mahfooz Haque, Jennifer Sharon Harris, Charlotte Anne Harrison, Tania Caroline St. Clair Hawthorn, Max Joseph Henderson, Trevor Hicks, Jennifer Hoblyn, Margaret Mary Horgan, Neil Richard Horn, Michael David Hunter, Susan Jane Jennings, Gillian Elizabeth Jess, Susan Lesley Wilson Jones (née Evans), Andras Gyula Juhasz, Saba Aslam Kaur, Afshan Naseem Khawaja, Ahmad Yassin Khouja, Siobhan Margaret King, Michael William Kingham, David Michael Kingsley, John Sik Nin Ko, David Ying Kit Lau, Karin Jessie Laudin, Che Kin Lee, Alison Mary Leech, Rachel Jane Lightstone, Caroline Ruth Linton, Arvind Kumar Lowe, Wing Cheong Lui, Wendy Forrest Macfarlane, Grenville Raymond Major, Nina Maria Malekottodjary, Karl Hemant Singh Marlowe, Richard Gavin Marriott, Mark David Martin, Timothy Paul
Matthews, Hamilton Stanley McBrien, Bernadette Margaret Elizabeth McCabe, David Cumming McCrindle, Celia Sarah Elizabeth McElwee, Joy Elizabeth Mcmahon, Ravi Mehrotra, Kenneth Mackinnon Mitchell, Deborah Anne Montgomery, Mary Patricia Moran, Colin George Morrison, Duart Murray Morrison, Simon David Mullins, Vijaya Murali, Tracey Louise Myton, Wai Man Stephen Ng, Aileen Ann O'Brien, Obed Obeng-Bekoe, Ajiro Nicola Omu, Smita Pandit, Jane Karen Perera, Mark Michael Picchioni, Jonathan Pimm, Konasale Muni Rajendra Prasad, Seena Praveen, Sundararajan Rajagopal, Bangaru Raju, Simona-Irina Rauta, Venumbaka Neelima Reddi, Suzanne Reeves, Jennifer Anne Reid, Michael Seamus Sean Reilly, Helen Margaret Reynolds, Lorna Alison Richards, Daniel Christopher Riordan, Caroline Ann Margaret Ritchie, Clare Jane Roberton, Florian Alexander (Tassilo) Ruths, Mona Salah Abdel-Baset Salem, Michael Peter Scully, Shahid Hamid Shabbir, Anand Sharma, Ruth Elizabeth Shaw, Rizwana Aqeel Siddique, Elita Smiley, John So, Marina Noelle Somers, Stephanie Marguerite Sommers, Robert Neil Spalding, Karim John Stavron, Joseph Anthony Stratford, Andre Strydom, Geetha Suryanarayan, Toby Gaunt Sutcliffe, Jane Katriona Sutherland, Catherine Taggart, Pamela Ann Taylor, Melanie Jayne Temple, John David Tendall, Thayalan Tibertius, Rebecca Jane Tipper, Rahul Tomar, Robert William Turner, Paul Umbrich, Girish Vaidya, Jakob Van der Boom, Apostolos Vourdas, Sally Walsh, Pamela Mary Walters, Anne Prior West, Stephen John Westgarth, Sian Nerys Weston, Michael Joseph Whelan, Jennifer Margaret White, Harvey Eugene Henry De La Vega Wickham, Paul Simon Williams, Sze Lai Shirley Wong.

\section{The Laughlin Prize}

The Laughlin Prize has been awarded to Dr Anne Cahill on the strength of her performance in the MRCPsych Part II Examination in Autumn 1999.

\section{The Standish-Barry Prize}

The Standish-Barry Prize has been awarded to Dr John Brady who was the candidate from an Irish Medical School who attained the highest mark in the MRCPsych Part II Examination in 1999. 
The College would like to take the opportunity to thank the following Clinical Centres and in particular the people who acted as Senior Organisers in Autumn 1999.

\section{Part I Clinical centres}

\begin{tabular}{lll} 
Senior organiser & Hospital & Centre \\
\hline Dr G. Loughrey & Knockbracken Healthcare Park & Belfast \\
Dr M. London & Addenbrooke's Hospital & Cambridge \\
Dr A. El-Komy & Forston Clinic & Dorset \\
Dr K. Gingell & Bushey Fields Hospital & Dudley \\
Dr K. Lloyd & Wonford House Hospital & Exeter \\
Dr R. Harrison & Dryden Road Day Hospital & Gateshead \\
Dr P. Jauhar & Parkhead Hospital & Glasgow \\
Dr U. Sivagama Sundari & St Cadoc's Hospital & Gwent \\
Professor M. Reveley & Glenfield General Hospital & Leicester \\
Dr A. Puranik & Invicta Community Care NHS Trust & Maidstone \\
Dr L. Hall & St Andrew's Hospital & Northampton \\
Dr P. Wilkinson & Warneford Hospital & Oxford \\
Dr J. Gallagher & Dykebar Hospital & Paisley \\
Dr K. Dudleston & Professional Training \& Development & Plymouth \\
Dr K. J. Cleary & Ealing, Hammersmith \& Fulham NHS Trust & Southall \\
Dr A. O. Yahia & Dorothy Pattison Hospital & Walsall \\
Dr A. Roberts & Queen Elizabeth II Hospital & Welwyn Garden City \\
Professor D. Jolley & New Cross Hospital & Wolverhampton
\end{tabular}

\section{Part II Clinical centres}

\begin{tabular}{lll}
\hline Senior organiser & Hospital & Centre \\
\hline Dr J. S. Callender & Royal Cornhill Hospital & Aberdeen \\
Dr E. M. O'Kane & Holywell Hospital & Antrim \\
Dr A. P. Roberts & Hergest Unit & Bangor \\
Dr K. Muthiah & Bedford Hospital & Bedford \\
Dr A. Patel & Queen Elizabeth Psychiatric Hospital & Birmingham \\
Dr J. Mathews & Parkwood & Blackpool \\
Dr N. Minton & Abraham Cowley Unit & Chertsey, Surrey \\
Dr J. G. Cunnane & Chesterfield \& North Derbyshire Royal Hospital & Chesterfield \\
Dr D. Walsh & St Stephen's Hospital & County Cork \\
Professor V. M. Mathew & Stone House Hospital & Dartford \\
Dr K. P. Rao & Derby Royal Infirmary & Derby \\
Professor T. J. Fahy & University College Hospital & Galway \\
Dr R. E. Hodgson & Lyme Brook Mental Health Centre & Keele \\
Dr M. Pierides & Lambeth Healthcare NHS Trust & London, SW9 \\
Dr V. Ramamurthy & University Hospital, Aintree & Liverpool \\
Dr F. Leslie & Fair Mile Hospital & Oxfordshire \\
Dr L. D. Mulgirigama & Surrey Oaklands NHS Trust & Oxted, Surrey \\
Dr P. Robinson & Royal Free Hospital & London, NW3 \\
Dr G. Moss & Meadowbrook & Salford \\
Dr M. L. Wesson & Hesketh Centre & Southport \\
Dr A. Bartlett & Springfield University Hospital & London, SW17 \\
Dr F. R. McKenzie & Airedale General Hospital & West Yorkshire \\
Dr D. Battin & Worcester Royal Infirmary & Worcester \\
\hline
\end{tabular}

